\title{
Medición de la gestión de la calidad universitaria: revisión bibliográfica*
}

\author{
Measurement of university quality management: \\ Bibliographic review
}

\section{Medição da gestão da qualidade universitária: Revisão bibliográfica}

Ximena Lucía Pedra:a Najar***

CORPOEDUCAD, Publimark Edninternational

Yuber Liliana Rodrígue* Rojas****

Convenio Universidad Santo Tomás - Icontec, Universitaria Agustiniana

Javier Pérez Juárez******

Universidad de Celaya - México

* Artículo de revisión. Muestra los resultados parciales de una investigación doctoral. DOI: http://dx.doi.org/10.15332/s2145-1389.2017.0001.01

** Doctora (c) en Administración. Magíster en Calidad y Gestión Integral. Especialista en Gestión de la Producción, la Calidad y la Tecnología. Especialista en Gerencia de Procesos, Calidad e Innovación. Microbióloga. Investigadora de la línea de Educación de la Corporación para la Administración y el Desarrollo Socioeducativo CORPOEDUCAD. Gerente general de Publimark Eduinternational. Correo electrónico: ximenapedraza@yahoo.com.

*** Doctora (c) en Administración. Magíster en Salud y Seguridad en el Trabajo. Fisioterapeuta. Líder de la línea de investigación en Gestión en Salud. Docente-investigador, convenio Universidad Santo Tomás - Icontec. Docente de posgrados de la Universitaria Agustiniana. Correo electrónico: yuberliliana@gmail.com.

**** Doctor en Administración. Magíster en Administración con especialidad en Calidad. Licenciado en Administración con especialidad en Mercadotecnia. Investigador en las líneas de Calidad, Productividad, Liderazgo, Desarrollo Organizacional, Mercadotecnia y Políticas Públicas Gubernamentales. Docente e investigador en la Universidad de Celaya y la Universidad Tecnológica del Centro de México. Correo electrónico: jperez@udec.edu.mx, javier.perez@utec-celaya.edu.mx, javier.perez@conagua.gob.mx. 


\section{RESUMEN}

Hablar de la calidad de la educación, es relacionarla con la excelencia y la competitividad de una comunidad educativa que tiene la necesidad de fortalecer e innovar sus procesos en un mundo de permanente cambio. La revisión bibliográfica realizada en ScienceDirect, ebrary, Emerald, EBSCO, Eric y Redalyc con un rango de búsqueda entre el año 2002 al 2016 permitió la consolidación de 11 dimensiones que integran 5 variables que inciden en la medición de la gestión de la calidad universitaria.

Palabras clave: calidad, gestión de la calidad, gestión de la calidad universitaria, medición de la calidad.

\section{ABSTRACT}

To talk about the quality of education is to relate it to the excellence and competitiveness of an educational community that has the need to strengthen and innovate its processes in a world of permanent change. The bibliographic review carried out in ScienceDirect, ebrary, Emerald, EBSCO, Eric and Redalyc with a search range between 2002 and 2016 allowed the consolidation of 11 dimensions that integrate 5 variables that influence the measurement of university quality management.

Keywords: Quality, quality management, university quality management, quality measurement.

\section{RESUMO}

Falar sobre a qualidade da educação é relacioná-la com a excelência e a competitividade de uma comunidade educativa com a necessidade de fortalecer e de inovar seus processos em um mundo de constante mudança.
A revisão bibliográfica feita em Science Direct, Ebrary, Emerald, Ebsco, Eric e Redalyc com um intervalo de pesquisa entre 0 ano 2002 até 2016 permitiu a consolidação de 11 dimensões que integram 5 variáveis que influenciam na Medição da gestão da qualidade universitária.

Palavras-chave: qualidade, gestão da qualidade, gestão da qualidade da universidade, medição da qualidade.

\section{INTRODUCCIÓN}

Para que las organizaciones se fortalezcan y sobrevivan a los tiempos de turbulencia, es necesario contar no solo con una alta productividad, sino que los productos y servicios cuenten con otras características de gran valor para los clientes o usuarios, tales como el precio, la oportunidad, la cobertura y la calidad. Esta última, a partir de la era de la globalización y en correspondencia con la teoría de las contingencias, se ha convertido en el elemento diferenciador y determinante para un servicio o un producto. Actualmente, la calidad adquiere un enfoque en términos de gestión, es decir, estableciendo directrices y orientaciones que permeen a las instituciones y de manera coordinada, son desplegadas y controladas en el marco de la eficacia, eficiencia y efectividad, para proyectarla en las organizaciones alrededor del mundo, en un momento único de integración de mercados, de competitividad, de innovación y de gestión del conocimiento.

Es por esto que al hablar de gestión de la calidad es pertinente establecerla como un conjunto de actividades en función general de la dirección que determina el horizonte en términos de calidad, sus objetivos, sus procesos e indicadores, con las responsabilidades correspondientes. Tiene fundamento en la satisfacción 
de las necesidades del cliente externo, sin desconocer que el cliente interno es muy importante para el logro de los propósitos de la organización, que se encuentran permeados por la calidad (Aldana et al., 2011).

La literatura sobre la gestión de la calidad de las organizaciones, se caracteriza por un fuerte interés por la teoría estadística, desarrollo de técnicas y, más recientemente, administración de recursos humanos y satisfacción del consumidor. Los clientes no siempre están satisfechos con la calidad y valor de los servicios que reciben. Esta situación, unida a una creciente competencia, provoca un frecuente conjunto de preocupaciones en los proveedores de servicios. El sector de los servicios de educación no está al margen de la competencia por los clientes, aumento de costes y críticas a la organización como un todo (Alvés, 2003).

Como cualquier organización, la educativa requiere de diversas herramientas para cumplir cabalmente su misión y sus propósitos. Ante ello, la disciplina administrativa cobra importancia singular dado que a través de su aplicación es posible transitar desde el establecimiento de objetivos y metas, y la determinación de las estrategias para lograrlos, hasta el establecimiento de sistemas de control para garantizar, hasta donde sea posible, la eficiencia de los resultados (Fernández y García, 2015). Los principales beneficios de la definición y aplicación de indicadores en el control de los procesos son: evaluar el desempeño del proceso con relación a las metas de mejoramiento definidas, mostrar tendencias, evaluar la efectividad y proveer señales oportunas para el mejoramiento (Serna, 2010).

De esta manera, a través de esta revisión bibliográfica se analiza el contexto de la gestión de la calidad universitaria, las variables y las dimensiones teóricas que pueden incidir en su medición. Esto permitirá que otros estudios utilicen esta información como insumo para generar instrumentos, válidos y confiables, que permitan medir la gestión de la calidad universitaria y su nivel de madurez.

\section{METODOLOGÍA}

La revisión de la literatura realizada sobre 143 artículos publicados entre 2002 y 2016, permitió identificar 25 de estos que le aportaban a la comprensión de la gestión de la calidad universitaria. El proceso de búsqueda se efectuó en ScienceDirect, ebrary, Emerald, EBSCO, Eric y Redalyc. Entre los términos clave utilizados en la búsqueda, se destacan: quality, quality management, quality management system, educational quality, quality measurement, quality service. La mayoría de los artículos detectados se han publicado entre 2012 y 2016, aspecto que evidenció que la medición de la gestión de la calidad universitaria es un tema de interés en la última década.

Estos documentos fueron revisados usando la técnica de análisis de contenido para detectar las dimensiones de la medición de la gestión de la calidad universitaria. Se determinó que 25 artículos cumplían con los requisitos de inclusión en el estudio. Además, se vincularon 3 normas internacionales, 6 libros y 3 fuentes adicionales que tienen relación directa con el constructo, objeto de investigación. El análisis de los documentos fue efectuado por dos investigadores de manera independiente, aspecto que brindó mayor objetividad en el proceso.

\section{RESULTADOS Y DISCUSIÓN}

Después del análisis del contenido de las referencias bibliográficas categorizadas, se identificaron 5 
variables que afectan la medición de la gestión de la calidad universitaria. A través de 11 dimensiones, estas variables proporcionan el estado de avance de una institución universitaria en términos de calidad en el servicio, como se presenta a continuación, en la tabla 1.

Tabla 1. Variables y dimensiones que influyen en la medición de la gestión de la calidad universitaria

\begin{tabular}{|c|c|c|c|}
\hline Variables & Dimensiones & $\begin{array}{l}\text { Número de fuentes } \\
\text { asociadas a la dimensión }\end{array}$ & $\begin{array}{l}\text { Bibliografía } \\
\text { de soporte }\end{array}$ \\
\hline \multirow{2}{*}{ 1. Ámbito } & a) Aseguramiento & 3 & (Skatulina, 2013); (Icontec, 2015); (Aldana et al., 2011). \\
\hline & b) Imagen & 2 & (Sebastián, 2002); (Téllez y Langebaek, 2014). \\
\hline \multirow{2}{*}{ 2. Visibilización } & c) Iniciativas & 3 & $\begin{array}{l}\text { (Consejo Nacional de Educación Superior CESU, 2014); } \\
\text { (Docampo, Egret y Cram, 2015); (Jarocka, 2015). }\end{array}$ \\
\hline & d) Adaptación & 3 & $\begin{array}{l}\text { (Collins y Park, 2015); (Holderness, Summers y Myers, } \\
\text { 2014); (Yudkevich, Altbach y Rumbley, 2015). }\end{array}$ \\
\hline \multirow[b]{2}{*}{ 3. Desempeño } & e) Liderazgo & 3 & $\begin{array}{l}\text { (Díez de Castro, Díez Martín y Vázquez, 2015); (Deloitte, } \\
\text { 2016); (Busetti y Dente, 2014). }\end{array}$ \\
\hline & $\begin{array}{l}\text { f) Seguimiento y } \\
\text { medición }\end{array}$ & 8 & $\begin{array}{l}\text { (Serna, 2010); (Álvarez, Chuairey y Pérez 2012); (Koni, } \\
\text { Zainal e Ibrahim, 2013); (Suprihatin y Bin, 2015); (Muller, } \\
\text { 2014); (Brochado, 2009); (Donlagic y Fazlic, 2015); } \\
\text { (Gómez y Sánchez, 2013). }\end{array}$ \\
\hline \multirow[t]{2}{*}{ 4. Recursos } & g) Humano & 4 & $\begin{array}{l}\text { (Bratianu y Pinzaru, 2015); (Toro, 2012); (Pedraja y } \\
\text { Rodríguez, 2014); (Ortiz, Funzy, Pérez y Velázquez, 2014). }\end{array}$ \\
\hline & h) Infraestructura & 3 & (Aldana et al., 2011); (Icontec, 2015); (Toro, 2012). \\
\hline \multirow{3}{*}{ 5. Estrategia } & i) Ejecución & 2 & $\begin{array}{l}\text { (Fernández, Sansevero y Lúquez, 2006); (Gutiérrez, } \\
\text { Ornelas y Mota, 2016). }\end{array}$ \\
\hline & j) Enfoque & 4 & $\begin{array}{l}\text { (Lumby y Foskett, 2016); (Ramírez, 2010); (Acosta y } \\
\text { Muñoz, 2007); (Gopalakrishnan, 2015). }\end{array}$ \\
\hline & k) Direccionamiento & 2 & (Álvarez, Torres y Chaparro, 2016); (Green, 2014). \\
\hline \multicolumn{2}{|c|}{ Número total de documentos } & $37^{*}$ & \\
\hline
\end{tabular}

Fuente: elaboración propia

* En total son 37 fuentes bibliográficas, puesto que 2 libros aportan en varias dimensiones. 


\section{1. Ámbito}

Este entorno global está cambiando constantemente y se caracteriza por el aumento de la competencia y la aparición de nuevos requisitos. A medida que las instituciones modernas de educación superior son sistemas no lineales complejos de múltiples niveles, dada su naturaleza, puesto que cada situación es particular, administradores de la educación, dedicados a la toma de decisiones estratégicas en un contexto muy complicado conformado por diversos factores contributivos, son determinantes debido a que sus decisiones están dirigidas a lograr una ventaja competitiva y la excelencia académica (Skatulina, 2013). De acuerdo con esto, se identifican elementos claves del ámbito institucional:

\section{a) Aseguramiento}

El enfoque en el aseguramiento, permite analizar la calidad más allá de la etapa de finalización de la prestación de un servicio, implica visualizar los procesos institucionales desde el inicio de la transformación, hasta la obtención de la confianza permanente y continua por parte del usuario del servicio. De esta manera, dentro del gran contexto de la calidad, se encuentra el compromiso permanente a proporcionar confianza en que se cumplirán los requisitos de productos y servicios asociados a la calidad que los caracteriza (Icontec, 2015). Esta etapa es la base del mejoramiento continuo como filosofía dentro de las instituciones educativas, puesto que crea la conciencia hacia la promesa permanente de prestar un servicio de acuerdo con lo establecido en los procesos definidos y de acuerdo con las necesidades determinadas por el receptor, con la proyección hacia la Administración por Calidad Total - Total Quality Management (TQM por sus siglas en inglés), pilar de todas las organizaciones que desean enfrentar un reto de cambio en cuanto a la forma de hacer las cosas, de trabajar de cara al cliente y de involucrar en cada acción de la organización la metodología del mejoramiento continuo (Aldana et al., 2011).

\section{b) Imagen}

Este elemento es evidente a partir del concepto de la visibilización de la educación superior que se ha enfocado hacia la internacionalización como una estrategia para el reconocimiento en sus funciones de calidad y pertinencia, de acuerdo con las características de cada institución y con las políticas públicas asociadas. La internacionalización de las Instituciones de Educación Superior (IES) se expresa a través de una doble vía. Por una parte, en la asimilación de la dimensión internacional en las funciones básicas de la docencia, la investigación y la extensión y por otra, en la ampliación de la oferta docente y de las capacidades existentes en la institución en un ámbito internacional (Sebastián, 2002).

La creciente importancia de la internacionalización en la educación superior ha propiciado, por un lado, que las IES establezcan prioridades y estrategias que les permitan alcanzar mayor presencia y visibilidad internacional en un mundo cada vez más globalizado; por otro lado, se ha convertido en un reto para los gobiernos nacionales y las entidades responsables del aseguramiento de la calidad dentro de los países en cuanto las posibilidades de inserción de programas e instituciones. En "lo internacional" dependen significativamente de los mecanismos de aseguramiento, evaluación y fomento a la calidad con que se cuente (Téllez y Langebaek, 2014).

\section{Visibilización}

En el campo de la educación, y a nivel superior, la visibilización se entiende como el reconocimiento de las instituciones por parte de la sociedad en relación 
con el ejercicio de sus funciones de calidad y pertinencia. Parte esta interpretación a partir de los procesos de acreditación institucional de las instituciones de educación superior en Colombia (Consejo Nacional de Educación Superior - CESU, 2014).

\section{c) Iniciativas}

La creciente influencia de la idea de universidades de clase mundial y el fenómeno asociado de las clasificaciones académicas internacionales, son cuestiones intrigantes para los análisis comparativos contemporáneos de la educación superior (Docampo, Egret y Cram, 2015). Muchos consideran que la clasificación de una universidad es muy importante no solo para el estudiante futuro, sino también para las propias universidades. Por lo tanto, existe una creciente necesidad de información fiable y transparente sobre las instituciones. Sin embargo, las universidades necesitan no solo datos estadísticos, sino también las herramientas que serán de utilidad en sus comparaciones y evaluaciones (Jarocka, 2015).

\section{d) Adaptación}

Ya sea en los sitios web, fuentes destacadas y documentación de "mejores universidades" del mundo o en más guías regionales y temáticas, ya sea a través de los sistemas de acreditación, métricas de revistas 0 índices internos; las tecnologías para la medición y clasificación de rendimiento académico no solo han creado nuevos imaginarios de reputación, también comenzó a formar de nuevo el comportamiento institucional en la búsqueda de un mejor rendimiento (Collins y Park, 2015). Se dice que los rankings y sus evidencias aumentan significativamente la cantidad de información disponible para al menos tres partes interesadas: los profesores, las instituciones de investigación y la industria. Esta última podría beneficiarse de dichas clasificaciones mediante la identificación de expertos en enseñanza para la consulta de los proyectos y la asignación de los fondos (Holderness, Summers y Myers, 2014).

Al mismo tiempo, hay que reconocer que no todas las universidades deben centrarse en un "nivel olímpico" de competencia, sino, más bien, debería centrarse en proporcionar el acceso, la educación de los alumnos, sirviendo y solucionando las necesidades locales y regionales en el marco de la denominada glocalización (Yudkevich, Altbach y Rumbley, 2015).

\section{Desempeño}

La teoría institucional nos indica que cuando las organizaciones se modernizan en su gestión, en su estructura y en su comportamiento, inician un proceso de cambio que las conduce a transformarse en instituciones (Díez de Castro, Díez Martín y Vázquez, 2015). Todo esto se realiza con base en las necesidades de cada empresa y dependiendo el tipo de industria en la que se encuentren (Deloitte, 2016).

\section{e) Liderazgo}

Los resultados de algunos estudios confirman el mal estado de la gestión de las administraciones universitarias y la existencia de inconvenientes inherentes de las administraciones de educación superior. Estas afirmaciones muestran que las dificultades específicas del sector (ambigüedad en los propósitos, de coproducción y la falta de recursos, entre otros), se suman a una negligencia generalizada de la importancia de una buena gestión por el liderazgo académico (Busetti y Dente, 2014).

\section{f) Seguimiento y medición}

Actualmente las organizaciones cuentan con diversas opciones para verificar su desempeño, ya sea a través 
de técnicas cualitativas, cuantitativas o mixtas. Desde el surgimiento de las técnicas para el control de la calidad a comienzos del siglo XX se han diseñado métodos que permiten medir y evaluar los procesos dentro de las organizaciones. Los principales beneficios de la definición y aplicación de indicadores en el control de los procesos son: evaluar el desempeño del proceso con relación a las metas de mejoramiento definidas, mostrar tendencias, evaluar la efectividad y proveer señales oportunas para el mejoramiento (Serna, 2010). Actualmente, se promueven investigaciones cuantitativas que permitan sistematizar los indicadores cuantitativos de calidad educativa para la América Latina y el Caribe (Álvarez, Chuairey y Pérez, 2012).

El modelo SERVQUAL ha sido aplicado como método de evaluación en diversas investigaciones relacionadas con la evaluación de la calidad del servicio de educación, como es el caso de las universidades palestinas, donde ha permitido identificar las oportunidades de mejoramiento que tienen para el fortalecimiento de la educación superior (Koni, Zainal e Ibrahim, 2013). Otros casos de aplicación se ven en asocio con el sistema de gestión de la calidad ISO-9001, aplicado en una institución de educación superior de Indonesia, donde se determinó el aporte integrado como elemento importante para el aseguramiento de la calidad universitaria (Suprihatin y Bin, 2015).

\section{Recursos}

El gobierno de la universidad se define como las formas constitucionales y procesos mediante los cuales las academias rigen sus asuntos. En una perspectiva más amplia, la gobernabilidad abarca las estructuras, las relaciones y los procesos por los cuales los niveles nacional e institucional se desarrollan, implementan y revisan las políticas para la educación. El manejo de los recursos comprende una compleja red, incluido el marco legislativo, las características de las instituciones y cómo se relacionan con todo el sistema, cómo el dinero se asigna a las instituciones y la forma en que son responsables de la forma en que se gasta, así como las estructuras y relaciones menos formales, las cuales dirigen e influencian el comportamiento (Bratianu y Pinzaru, 2015). Los recursos financieros, si bien no son componentes directos de procesos académicos, son los que permiten la adquisición de los demás recursos y deben ser tenidos en cuenta en cualquier estudio de la operación de una institución de educación superior (Toro, 2012).

\section{g) Humano}

En el campo de la educación, uno de los aspectos fundamentales para generar una formación adecuada es contar con líderes que ejerzan un rol central en la formación universitaria y en la inspiración y amor por el trabajo a las nuevas generaciones. En consecuencia, en la educación superior se ha comprobado la importancia del estilo de liderazgo transformacional para generar una visión compartida entre el líder y el equipo, junto al compromiso, colaboración y una estimulación mutua, basada en el respeto por el líder, en su carisma y en su capacidad para motivar a sus seguidores (Pedraja y Rodríguez, 2014).

\section{h) Infraestructura}

La estructura organizacional por procesos facilita, por su enfoque, la implementación de la cultura de la calidad, pues se basa en la conformación de equipos de trabajo para el logro de resultados que aseguren satisfacer las necesidades de los clientes (internos y externos). La reorganización de la estructura permite dar una respuesta más eficaz a los cambios que se producen por las necesidades del mercado, siendo una de las claves propuestas para lograr la calidad y hacer a una 
empresa más competitiva (Aldana et al., 2011). El funcionamiento exitoso de una organización dependerá de la claridad y determinación con las que identifique sus procesos para la operación y de la organización y orientación que se les otorgue. La comprensión y gestión de los procesos interrelacionados como un sistema contribuyen a la eficacia y eficiencia de la organización en el logro de sus resultados previstos. Este enfoque permite a la organización controlar las interrelaciones e interdependencias entre los procesos del sistema, de modo que se pueda mejorar el desempeño global de la organización (Icontec, 2015).

\section{i) Ejecución}

La teoría general de los sistemas constituye un eje orientador para explicar el comportamiento heurístico, interdisciplinar e integrador de los procesos gerenciales que facilitan la interacción docente-alumno en el aula; puesto que la misma ofrece una perspectiva que implica estudiar un fenómeno en toda su complejidad al tomar en cuenta las partes, no en forma aislada sino a partir de la estructura del todo; tal es el caso del aula 0 ambiente de aprendizaje, sistema conformado por los elementos básicos: entrada, proceso y salida (Fernández, Sansevero y Lúquez, 2006).

\section{Estrategia}

Competitividad ya no es un término exclusivamente utilizado en el sector industrial o empresarial, ahora no solo se exigen organizaciones competitivas, sino que además los individuos que laboran en esas organizaciones deben ser altamente competitivos y poseer un capital intelectual sólido que permita desarrollar innovación continua y vanguardista para ubicar a sus empresas dentro de las mejores a nivel global (Lumby y Foskett, 2016).

\section{j) Enfoque}

Está en manos de los gobernantes, directores, gerentes, empresarios y gestores de las organizaciones y de las empresas, poner en práctica los principios de la administración científica. Hay que acabar con el subdesarrollo y entender, de una vez por todas, que el problema de nuestros países, en primer lugar, es un problema de bajos niveles de dirección, de gestión y de gerencia, esto es, de baja capacidad administrativa que tampoco permite el progreso y avance de los procesos educativos de los pueblos. Esta baja capacidad administrativa que incluye en la distorsión de valores sociales y propicia la corrupción y la inmoralidad gerencial (Ramírez, 2010).

\section{k) Direccionamiento}

En el ámbito universitario, el Liderazgo Educativo, a través de sus tres variables: Liderazgo Institucional, Liderazgo Directivo y Liderazgo Pedagógico, permite la administración adecuada de las instituciones involucrando a todos los actores de la comunidad escolar bajo un propio direccionamiento (Álvarez, Torres y Chaparro, 2016).

\section{CONCLUSIONES}

De acuerdo con las fuentes bibliográficas consultadas, es posible medir la gestión de la calidad a través de 5 variables: ámbito, visibilización, desempeño, recursos y estrategia y once dimensiones. Estos resultados están alineados con lo descrito por Icontec (2010), que a través del modelo de gestión para el logro del éxito sostenido de las organizaciones, permite cuantificar la gestión de la calidad en el contexto universitario. Medir la gestión a través del referencial ISO-9004 permite que las IES avancen hacia la excelencia en el contexto de la calidad en la prestación del servicio. 
A nivel mundial, se están realizando exploraciones e investigaciones relacionadas de acuerdo con el contexto cultural y legislativo de cada nación, una de las cuestiones de fondo en valores de la organización actual del discurso es la falta de acuerdo sobre los descriptores relacionados con el fenómeno, el cual es extraordinariamente complejo (Muller, 2014). Dichos componentes deben ser vistos como parte de un sistema que involucra personas, procesos y recursos que se le podría denominar sistema de la institución de educación superior (Toro, 2012).

El instrumento SERVQUAL, a pesar de las críticas por una variedad de autores, todavía parece ser el modelo más práctico para la medición de la calidad de los servicios disponibles en la literatura, por lo tanto las expectativas deben ser considerados al evaluar la calidad del servicio en la educación superior (Brochado, 2009). En otro estudio, la aplicación se hizo en una facultad particular, la de Economía, con resultados orientados hacia la mejora institucional (Donlagic y Fazlic, 2015). La información generada a partir de la aplicación de los métodos elegidos o desarrollados, permite a la institución la identificación de oportunidades de mejora continua, sirviendo como insumo para el proceso de planificación estratégica, y en general para el desarrollo de planes de acción y el cumplimiento de los objetivos propuestos (Acosta y Muñoz, 2007).

En el sector educativo se han realizado estudios que han permitido identificar los parámetros primordiales y los componentes para evaluar la gestión académica (Gómez y Sánchez, 2013). En Sudáfrica, la aplicación se hizo con el objetivo de identificar la efectividad de la prestación del servicio con la identificación de planes de mejoramiento especiales (Green, 2014). En el sector de la educación, la retroalimentación de los estudiantes es muy importante (Gopalakrishnan, 2015). En la Universidad de Holguín (Cuba) se analizó el nivel de integración de los procesos a partir de la gestión de la calidad descrita en la norma ISO-9001, dando como resultado que la comunicación institucional, la gestión de la actividad científico estudiantil, el trabajo docente y la formación académica, contribuyen a lograr mayor efectividad en el proceso de toma de decisiones al realizar un análisis integrado en la universidad (Ortiz, Funzy, Pérez y Velázquez, 2014). Así mismo, los resultados han combinado de forma ponderada el interés por las personas con la importancia de los recursos, de los procesos y de los resultados (Sánchez, Pacheco y Gándara, 2016).

En resumen, las dimensiones y variables identificadas a partir de la revisión bibliográfica permiten establecer los elementos que inciden en la medición de la gestión de la calidad que se está llevado a cabo en las instituciones universitarias, así como determinar en qué medida se ha apropiado en todos los niveles organizacionales.

Al medir la gestión de la calidad universitaria se está consolidando una línea base del mejoramiento continuo institucional, el cual permite, desde un análisis del contexto, identificar las iniciativas para potenciar los recursos con los que cuenta y de esta manera garantizar que la calidad sea una forma de pensar y una herramienta para consolidarse como ejemplo de una organización para la sociedad.

La medición de la gestión de la calidad universitaria se convierte en una temática de interés para ser explorada con más detenimiento, puesto que los tiempos globalizados hacen que el servicio educativo se caracterice por los componentes que le generen valor y estos no solo se determinan a partir de reconocimientos como la certificación, acreditación o premios de excelencia, sino de una gestión sistemática, controlada y mejorada que permita la generación de competencias integrales en los estudiantes en un mercado laboral, exigente y competitivo. 


\section{REFERENCIAS}

Aldana, L., Álvarez, M., Bernal, C., Díaz, M., González, C., Galindo, Ó., y Villegas, A. (2011). Administración por calidad. México: Editorial Alfaomega.

Acosta, A., y Muñoz, M. (2007). La autoevaluación: una iniciativa para la gestión de la calidad en educación superior. Ingeniería Industrial, 28(3), 35-39.

Álvarez, J., Torres, A., y Chaparro, E. (2016). Diagnóstico del liderazgo educativo en las Instituciones de Educación Superior del Valle de Toluca. Revista de Investigación Educativa, 34(1), 51-68.

Álvarez, Y., Chuairey, L., y Perez, J. (2012). Modelos matemáticos para el pronóstico de indicadores cuantitativos que miden la calidad de la educación superior en la carrera de agronomía de la Universidad Agraria de la Habana. Investigación Operacional, 33(2), 173-180.

Alvés, G. (2003). Marketing en los servicios de educación: modelos de percepción de calidad. Tesis doctoral. Madrid: Universidad Complutense de Madrid.

Bratianu, C., \& Pinzaru, F. (2015). University Governance as a Strategic Driving Force. European Conference on Management, Leadership \& Governance, 9. Lisbon: Academic Conferences \& Publishing International Ltd.

Brochado, A. (2009). Comparing alternative instruments to measure service quality in higher education. Quality Assurance in education, 17(2), 174-190.

Busetti, S., \& Dente, B. (2014). Focus on the finger, overlook the moon: the introduction of performance management in the administration of Italian universities. Journal of Higher Education Policy and Management, 36(2), 225-237.
Collins, F., \& Park, G. (2015). Ranking and the multiplication of reputation: reflections from the frontier of globalizing higher education. Higher Education, 72(1), 115-129.

Consejo Nacional de Educación Superior - CESU. (2014). Lineamientos para la acreditación institucional. Acuerdo 03 de 2014. Bogotá: Consejo Nacional de Educación Superior CESU.

Deloitte. (21 de abril de 2016). Implementación, organización y desafios. Consultoría en Capital Humano. Recuperado de https://www2.deloitte.com/content/dam/Deloitte/co/Documents/human-capital/ brochure\%20Consultor\%C3\%ADa.pdf

Díez de Castro, E., Díez Martín, F., y Vázquez, A. (2015). Antecedentes de la institucionalización de las organizaciones. Cuadernos de gestión, 15(1).

Docampo, D., Egret, D., \& Cram, L. (2015). The effect of university mergers on the Shanghai ranking. Scientometrics, 104(1), 175-191.

Donlagic, S., \& Fazlic, S. (2015). Quality assessment in higher education using the servqual model. Management Journal of Contemporary Management Issues, 20(1), 39-57.

Fernández, M., y García, J. (2015). Algunos fundamentos educativos y administrativos para la propuesta de creación de la Línea de Generación y Aplicación del Conocimiento: gestión educativa en el Instituto de Investigaciones y Estudios Superiores de las Ciencias Administrativas. Ciencia Administrativa, (2), 1-8.

Fernández, O., Sansevero, I., y Lúquez, P. (2006). Un modelo de gerencia de los aprendizajes en el aula bajo una perspectiva sistémica. Encuentro Educacional, 13(3). 
Gómez, H., y Sánchez, V. (2013). Indicadores cualitativos para la medición de la calidad en la educación. Educación y Educadores, 16(1), 9-24.

Gopalakrishnan, G. (2015). How to apply academic supply chain management: the case of an international university: the case of an international university. Management: Journal of Contemporary Management Issues, 20(1), 207-221.

Green, P. (2014). Measuring service quality in higher education: a South African case study. Journal of International Education Research, 10(2), 131-142.

Gutiérrez, J., Ornelas, M., y Mota, M. (2016). Modelo de calidad para instituciones de educación superior. Mercados y negocios (1665-7039), 13, 113-139.

Holderness, D., Summers, S., \& Myers, N. (2014). Accounting education research: ranking institutions and individual scholars. Issues in Accounting Education, 29(1), 87-115.

Instituto Colombiano de Normas Técnicas y Certificación - Icontec. (2015). Norma técnica colombiana NTC ISO-9000. Sistema de gestión de la calidad. Fundamentos y vocabulario. Bogotá: Icontec.

Instituto Colombiano de Normas Técnicas y Certificación - Icontec. (2015). Norma técnica colombiana NTC ISO-9000. Sistema de gestión de la calidad. Requisitos. Bogotá: Icontec.

Instituto Colombiano de Normas Técnicas y Certificación - Icontec. (2010). Norma técnica colombiana NTC ISO-9004. Gestión para el éxito sostenido de una organización. Enfoque de gestión de la calidad. Bogotá: Icontec.

Jarocka, M. (2015). Transparency of university rankings in the effective management of university. Business, management and education, 13(1), 64-75.
Koni, A., Zainal, K., \& Ibrahim, M. (2013). An assessment of the services quality of Palestine higher education. International Education Studies, $6(2), 33-48$.

Lumby, J., y Foskett, N. (2016). Internationalization and culture in higher education. Educational Management Administration \& Leadership, 44(1), 95-111.

Mueller, R. (2014). A general model of organizational values in educational administration. Educational Management Administration \& Leadership, 42(5), 640-656.

Ortiz, A., Funzy, J., Pérez, M., y Velázquez, R. (2014). La gestión integrada de los procesos en universidades. Procedimiento para su evaluación. Ingeniería Industrial, 36(1), 91-103.

Pedraja, L., y Rodríguez, E. (2014). Formación, liderazgo y un caso real de líder transformacional en una universidad. Diálogo Andino, 44, 3-4.

Ramírez, C. (2010). Fundamentos de Administración. Bogotá: Ecoe Ediciones.

Sebastián, J. (2002). La dimension internacional en los procesos de evaluacion y acreditacion de la educacion superior. Madrid, España: Centro de Información y Documentación Científica. Consejo Superior de Investigaciones Científicas.

Serna, H. (2010). Gerencia estratégica. Bogotá, Colombia: 3R Editores.

Skatulina, Y. (2013). Management of the educational environment: the context in which strategic decisions are made. Procedia-social and behavioral sciences, 99, 1054-1062. 
Suprihatin, K., \& Bin, H. (2015). Development design model of academic quality assurance at private islamic University Jakarta Indonesia. Higher Education Studies, 5(4), 6-29.

Téllez, F., y Langebaek, C. (2014). Internacionalisación y calidad en Colombia: el rol estratégico de la acreditación en el fortalecimiento de los procesos de internacionalización de la educación superior. Bogotá: Multiimpresos SAS.

Toro, J. (2012). Gestión interna de la calidad en las instituciones de educación superior. RIL Editores.

Yudkevich, M., Altbach, P., \& Rumbley, L. (2015). Global university rankings: the "Olympic Games" of higher education? Prospects, 45(4), 411-419. 